\title{
AXISYMMETRIC AND NONAXISYMMETRIC BUCKLED STATES OF A SHALLOW SPHERICAL CAP*
}

\author{
BY
}

\author{
FRANK E. BAGINSKI
}

The George Washington University, Washington, D.C.

1. Introduction. In this paper we will study the buckling of a thin elastic shallow spherical cap, simply supported at its edge, and subjected to a constant centrally directed external pressure.

The experimental evidence in [5] and [6], for a complete spherical shell, indicates that a buckled state of a spherical shell may possess various degrees of symmetry. The most symmetric buckled state observed is that of a circular dimple, while the least symmetric state observed is that of a pear-shaped dimple with one plane of reflectional symmetry. We will show that when the cap is shallow, there exist stable axisymmetric solutions. In addition, we will show the existence of a variety of other unstable solutions which bifurcate from the unbuckled state of the shell.

Various models have been used to study the axisymmetric buckling of a spherical shell (see [2], [12], [20]). The Von Kármán model or equivalent versions have been used to study the buckling of a shallow shell (see [3], [4], [19], [25]). We shall use the "lowest order interior shell equations" of John $[9 ; 10]$ in the work presented here. Because they are derived with no a priori assumptions on the size of the displacements of the shell or the initial curvature, $K$, of the shell, the John equations provide an accurate mathematical model which is an improvement over the previously mentioned ones. The John equations (or equivalent versions) have been used in [13] and [15] to describe the nonaxisymmetric buckling of a complete spherical shell.

In Section 2, a "shallow cap" version of the John equations is developed. In Section 3, it is shown that the problem of determining the buckled states of a simply supported spherical cap reduces to solving the equation,

$$
w-\lambda A w+\tau^{2} A^{2} w+\tau Q(w)+C(w)=0,
$$

for $w \in \mathscr{H}$, where $\mathscr{H}$ is an appropriate Hilbert space. Determining the solutions of $(*)$ is complicated by the fact that nonaxisymmetric solutions of $(*)$ generate oneparameter families of solutions, so that solutions are not generally isolated. In Section 4 , the experimental work of [5] and [6] is used to motivate a way of avoiding this problem. Once a certain symmetry assumption is made, axisymmetric and nonaxisymmetric solutions of $(*)$ can be determined by applying the Liapunov-Schmidt method (see Theorem 1 in Section 5) on a "smaller" Hilbert space, $\mathscr{W}$, contained in 
$\mathscr{H}$. The solutions in $\mathscr{W}$ can then be used to generate solutions in $\mathscr{H}$. The stability of these solutions is discussed in Section 6. The main result of Section 6 is Theorem 2, where we show the existence of stable axisymmetric buckled states of a spherical cap. In particular, if $\left|\lambda-\lambda_{c}\right|$ is sufficiently small, then there exist two nontrivial solutions for certain values of $\lambda<\lambda_{c}$ (one is stable, the other is unstable); for certain values of $\lambda>\lambda_{c}$, there exist two stable nontrivial solutions. A list of symbols is contained at the end of this paper.

2. The interior shell equations of John. The behavior of an elastic shell under the influence of a centrally directed pressure is described by the solutions of a system of second-order nonlinear partial differential equations posed by John $[9 ; 10]$. The lowest order interior shell equations of John are given by:

$$
\begin{gathered}
\left.\frac{1}{E h} n_{\alpha}^{\alpha}\right|_{\beta} ^{\beta}+\tilde{b}_{\alpha}^{\alpha} \rho_{\beta}^{\beta}-\tilde{b}_{\beta}^{\alpha} \rho_{\alpha}^{\beta}+\frac{1}{2}\left(\rho_{\alpha}^{\alpha} \rho_{\beta}^{\beta}-\rho_{\beta}^{\alpha} \rho_{\alpha}^{\beta}\right)=0, \\
\left.\rho_{\beta}^{\alpha}\right|_{\mu}-\left.\rho_{\mu}^{\alpha}\right|_{\beta}=0, \\
\left.\frac{E h^{3}}{12\left(1-\nu^{2}\right)} \rho_{\alpha}^{\alpha}\right|_{\beta} ^{\beta}-\left(\tilde{b}_{\beta}^{\alpha}+\rho_{\beta}^{\alpha}\right) n_{\alpha}^{\beta}=-\hat{P}, \\
\left.n_{\beta}^{\alpha}\right|_{\alpha}=0 .
\end{gathered}
$$

Equations (2.1)-(2.4) are equivalent to the following system developed by Koiter in [16]:

$$
\begin{gathered}
\tilde{\varepsilon}^{\alpha \sigma} \tilde{\varepsilon}^{\beta \mu}\left[\left.\gamma_{\alpha \beta}\right|_{\sigma \mu}+\tilde{b}_{\alpha \beta} \rho_{\sigma \mu}+\frac{1}{2} \rho_{\alpha \beta} \rho_{\sigma \mu}\right]=0, \\
\left.\tilde{\varepsilon}^{\alpha \sigma} \tilde{\varepsilon}^{\beta \mu} \rho_{\sigma \mu}\right|_{\beta}=0, \\
\left.m^{\alpha \beta}\right|_{\alpha \beta}-\left(\tilde{b}_{\alpha \beta}+\rho_{\alpha \beta}\right) n^{\alpha \beta}=-\hat{P}, \\
\left.n^{\alpha \beta}\right|_{\beta}=0, \\
\gamma_{\alpha \beta}=\frac{1}{E h}\left[(1+\nu) n_{\alpha \beta}-\nu \tilde{a}_{\alpha \beta} n_{\sigma}^{\sigma}\right], \\
m^{\alpha \beta}=E h^{3} \gamma^{2}\left[(1-\nu) \rho^{\alpha \beta}+\nu \tilde{a}^{\alpha \beta} \rho_{\sigma}^{\sigma}\right],
\end{gathered}
$$

where $h$ is the thickness of the shell, $E$ is Young's modulus, $\nu$ is Poisson's ratio, $\gamma^{2}=\left[12\left(1-\nu^{2}\right)\right]^{-1}$, and $\hat{P}$ is the normal load $(\hat{P}$ is positive when the pressure is directed inward).

The unknowns in (2.1)-(2.4) are four symmetric tensors: $\gamma_{\alpha \beta}, \rho_{\alpha \beta}, n^{\alpha \beta}$ and $m^{\alpha \beta}$. $\gamma_{\alpha \beta}$ is the strain tensor and measures the difference between the first fundamental form, $\bar{a}_{\alpha \beta}$, of the deformed shell and the first fundamental form, $\tilde{a}_{\alpha \beta}$, of the undeformed shell, i.e.,

$$
\gamma_{\alpha \beta}=\frac{1}{2}\left(\bar{a}_{\alpha \beta}-\tilde{a}_{\alpha \beta}\right)
$$

$\rho_{\alpha \beta}$ is the change of curvature tensor and measures the difference between the second fundamental form, $\bar{b}_{\alpha \beta}$, of the deformed shell and the second fundamental form, $\tilde{b}_{\alpha \beta}$, of the undeformed shell, i.e.,

$$
\rho_{\alpha \beta}=\bar{b}_{\alpha \beta}-\tilde{b}_{\alpha \beta}
$$


The symbol, " $\left.\right|_{\alpha}$ ", denotes covariant differentiation with respect to the metric tensor, $\tilde{a}_{\alpha \beta} . m^{\alpha \beta}$ is the tensor of stress couples and $n^{\alpha \beta}$ is the tensor of stress resultants. The geometric quantities, $\gamma_{\alpha \beta}$ and $\rho_{\alpha \beta}$, are related to the physical quantities, $m^{\alpha \beta}$ and $n^{\alpha \beta}$, through the constitutive relations, (2.9) and (2.10).

Let $S_{R}$ denote the sphere of radius $R$ in $\mathbf{R}^{3}$. Let $\left(x_{1}, x_{2}, x_{3}\right)$ be a cartesian coordinate system with origin at the center of the sphere. If $X \in S_{R}$, then $X$ may be parametrized by $X(\theta, \phi)=R(\cos \phi \sin \theta, \sin \phi \sin \theta, \cos \theta)$, for $0 \leq \theta \leq \pi$ and $0 \leq \phi \leq 2 \pi$. Let $S_{R}(\Omega)$ denote the subset of $S_{R}$ given by

$$
S_{R}(\Omega)=\left\{X \mid X(\theta, \phi) \in S_{R},(\theta, \phi) \in \Omega\right\}
$$

where

$$
\Omega=\left\{(\theta, \phi) \mid 0 \leq \theta \leq \theta_{0}<\pi / 2,0 \leq \phi \leq 2 \pi\right\} .
$$

The boundary of $S_{R}(\Omega)$ is the set,

$$
S_{R}(\partial \Omega)=\left\{X \mid X(\theta, \phi) \in S_{R},(\theta, \phi) \in \partial \Omega\right\},
$$

where

$$
\partial \Omega=\left\{(\theta, \phi) \mid \theta=\theta_{0}, 0 \leq \phi \leq 2 \pi\right\} .
$$

Thus, $S_{R}(\Omega)$ is a spherical cap with opening $2 \theta_{0}$. For convenience, hereafter we will refer to " $\Omega$ " as a spherical cap with boundary, " $\partial \Omega$ "; it is clear from the definition of $\Omega$ how this statement should be interpreted.

The first and second fundamental forms in $S_{R}\left(\right.$ and $\left.S_{R}(\Omega)\right)$ are given by

$$
\tilde{a}_{\alpha \beta}=\left[\begin{array}{cc}
R^{2} & 0 \\
0 & R^{2} \sin ^{2} \theta
\end{array}\right] \text { and } \tilde{b}_{\alpha \beta}=-\frac{1}{R} \tilde{a}_{\alpha \beta},
$$

respectively. In computing $\tilde{b}_{\alpha \beta}$, we use $\mathbf{r}=R^{-1} X$ as the unit normal to $S_{R}(\Omega)$. The alternating tensor, $\tilde{\varepsilon}_{\alpha \beta}$, is given by

$$
\tilde{\varepsilon}_{\alpha \beta}=\left[\begin{array}{cc}
0 & \tilde{a}^{1 / 2} \\
-\tilde{a}^{1 / 2} & 0
\end{array}\right]
$$

where $\tilde{a}=\operatorname{det}\left(\tilde{a}_{\alpha \beta}\right)$. If $R=1$, then the corresponding tensors on $S_{1}(\Omega)$ will be denoted by $a_{\alpha \beta}, b_{\alpha \beta}, \varepsilon_{\alpha \beta}$. If we let $\theta_{1}=\theta$ and $\theta_{2}=\phi$, then the Christoffel symbols on $S_{R}$ are given by

$$
\Gamma_{11}^{1}=\Gamma_{11}^{2}=\Gamma_{21}^{1}=\Gamma_{12}^{1}=\Gamma_{22}^{2}=0, \quad \Gamma_{22}^{1}=-\sin \theta_{1} \cos \theta_{1}, \quad \Gamma_{12}^{2}=\Gamma_{21}^{2}=\cot \theta_{1} \text {. }
$$

For $w=w\left(\theta_{1}, \theta_{2}\right)$, the covariant derivatives of $w$ are given by $\left.w\right|_{\alpha}=w_{, \alpha}$ and $\left.w\right|_{\alpha \beta}$ where

$$
\begin{aligned}
& \left.w\right|_{11}=w_{, 11}, \\
& \left.w\right|_{12}=w_{, 12}-\cot \theta_{1} w_{, 2}, \\
& \left.w\right|_{22}=w_{, 22}+\sin \theta_{1} \cos \theta_{1} w_{, 1} .
\end{aligned}
$$

When the undeformed shell is a sphere of radius $R$, the general solutions of (2.6) and (2.8) are given by (see Truesdell [23])

$$
\begin{aligned}
& \rho_{\alpha \beta}=\left.W\right|_{\alpha \beta}+K W \tilde{a}_{\alpha \beta}, \\
& n^{\alpha \beta}=\left.\tilde{\varepsilon}^{\alpha \sigma} \tilde{\varepsilon}^{\beta \mu} F\right|_{\sigma \mu}+K F \tilde{a}^{\alpha \beta},
\end{aligned}
$$


where $K=1 / R^{2}$ is the Gauss curvature of the undeformed sphere. $W$ and $F$ are referred to as the generalized curvature function and stress function, respectively. Using (2.11) and (2.12) along with the constitutive relationships, (2.9) and (2.10), one may rewrite equations (2.5) and (2.7) as (see Knightly and Sather [13])

$$
\begin{aligned}
& (E h)^{-1}\left(\tilde{\Delta}^{2} F+2 K \tilde{\Delta} F\right)-\frac{1}{R}(\tilde{\Delta} W+2 K W) \\
& \quad+\left.\frac{1}{2}\left(\left.\tilde{\varepsilon}^{\alpha \lambda} \tilde{\varepsilon}^{\beta \mu} W\right|_{\lambda \mu} W_{, \alpha}\right)\right|_{\beta}+\frac{1}{2}\left(K \tilde{a}^{\alpha \beta} W_{, \alpha} W_{, \beta}+2 K W \tilde{\Delta} W+2 K^{2} W^{2}\right)=0,
\end{aligned}
$$

$$
\begin{aligned}
& E h^{3} \gamma^{2}\left(\tilde{\Delta}^{2} W+2 K \tilde{\Delta} W\right)+\frac{1}{R}(\tilde{\Delta} F+2 K F) \\
& \quad-\left.\left(\left.\tilde{\varepsilon}^{\alpha \lambda} \tilde{\varepsilon}^{\beta \mu} F\right|_{\lambda \mu} W_{, \alpha}\right)\right|_{\beta}-\left(K \tilde{a}^{\alpha \beta} W_{, \alpha} F_{, \beta}+K(F \tilde{\Delta} W+W \tilde{\Delta} F)+2 K^{2} F W\right)=-\hat{P} .
\end{aligned}
$$

Equations (2.13) and (2.14) have been studied in [13] and [15] for the case of a full sphere (i.e., without boundary).

Equations (2.13) and (2.14) can be rewritten in the form,

$$
\begin{gathered}
\frac{1}{E h} \tilde{\Delta}(\tilde{\Delta} F+2 K F)-\frac{1}{R}(\tilde{\Delta} W+2 K W)+\frac{1}{2}\{W, W\}_{R}=0, \\
E h^{3} \gamma^{2} \tilde{\Delta}(\tilde{\Delta} W+2 K W)+\frac{1}{R}(\tilde{\Delta} F+2 K F)-\{W, F\}_{R}=-\hat{P},
\end{gathered}
$$

where $\tilde{\Delta}$ denotes the Laplacian on $S_{R}(\Omega)$,

$$
\{U, V\}_{R}=\left.\left(\left.\tilde{\varepsilon}^{\sigma \kappa} \tilde{\varepsilon}^{\beta \mu} U\right|_{\kappa \mu} V_{, \sigma}\right)\right|_{\beta}+K \tilde{a}^{\sigma \beta} U_{, \sigma} V_{, \beta}+K(U \tilde{\Delta} V+V \tilde{\Delta} U)+2 K^{2} U V,
$$
and $K=1 / R^{2}$.

If we set $F=E h^{3} \gamma^{2} \hat{f}, W=h \gamma w$, and $\hat{P}=p / R$ in (2.15) and (2.16) we obtain

$$
\begin{gathered}
\tilde{\Delta}^{2} \hat{f}+2 K \tilde{\Delta} \hat{f}-\frac{1}{h \gamma R}(\tilde{\Delta} w+2 K w)+\frac{1}{2}\{w, w\}_{R}=0, \\
\tilde{\Delta}^{2} w+2 K \tilde{\Delta} w+\frac{1}{h \gamma R}(\tilde{\Delta} \hat{f}+2 K \hat{f})-\{w, \hat{f}\}_{R}=-\frac{p}{R E h^{4} \gamma^{3}} .
\end{gathered}
$$

If simply supported boundary conditions are imposed on $(w, \hat{f})$, then

$$
w=\tilde{\Delta} w=0 \text { on } \partial \Omega,
$$

and

$$
\hat{f}=\frac{-p R^{2}}{2 E h^{3} \gamma^{2}}, \quad \Delta \hat{f}=0 \quad \text { on } \partial \Omega .
$$

Let $\hat{f}=-f_{0}+f$, where $f_{0}$ is constant on $\Omega$. If $(w, f)=(0,0)$ is a solution for all $p \in \mathbf{R}^{1}$, then $f_{0}$ must be chosen such that

$$
f_{0}=\frac{p R^{2}}{2 E h^{3} \gamma^{2}} \text {. }
$$

Prior to buckling, the cap is assumed to remain spherical (i.e., $(w, f)=(0,0)$ ). We call $(w, \hat{f})=\left(0,-\left(p R^{2}\right) /\left(2 E h^{3} \gamma^{2}\right)\right)$ the trivial solution. A nontrivial solution is a pair 
$(w, f) \neq(0,0)$ that satisfies

$$
\begin{aligned}
& 0=\tilde{\Delta}^{2} f+2 K \tilde{\Delta} f-\frac{1}{h \gamma R}(\tilde{\Delta} w+2 K w)+\frac{1}{2}\{w, w\}_{R}, \\
& 0=\tilde{\Delta}^{2} w+2 K \tilde{\Delta} w+\frac{1}{h \gamma R}(\tilde{\Delta} f+2 K f)+\frac{p}{E h^{3} \gamma^{2}}(\tilde{\Delta} w+2 K w)-\{w, f\}_{R}, \\
& w=0, \quad \tilde{\Delta} w=0 \quad \text { on } \partial \Omega
\end{aligned}
$$

and

$$
f=0, \quad \tilde{\Delta} f=0 \quad \text { on } \partial \Omega .
$$

It is convenient to rewrite the above equations in terms of polar coordinates. In the following, $\rho_{0}=R \sin \theta_{0}$ is fixed. If we multiply (2.23)-(2.24) by $\rho_{0}^{4}$ and (2.25)-(2.26) by $\rho_{0}^{2}$, and then substitute $s=(R \sin \theta) / \rho_{0}$ into the resulting equations we obtain

$$
\begin{aligned}
& 0=\Delta_{s}^{2} f+\frac{1}{2}[w, w]_{s}-\tau \Delta_{s} w+\sin ^{2} \theta_{0} M(w, f), \\
& 0=\Delta_{s}^{2} w-[w, f]_{s}+\tau \Delta_{s} f+\lambda \Delta_{s} w+\sin ^{2} \theta_{0} N(w, f),
\end{aligned}
$$

where

$$
\begin{aligned}
& w=0, \quad \Delta_{s} w-\sin ^{2} \theta_{0} D_{s} w=0 \quad \text { on } \partial \Omega, \\
& f=0, \quad \Delta_{s} f-\sin ^{2} \theta_{0} D_{s} f=0 \quad \text { on } \partial \Omega, \\
& \tilde{\Delta} w=\frac{1}{\rho_{0}^{2}}\left[\Delta_{s} w-\sin ^{2} \theta_{0} D_{s} w\right] \\
& \Delta_{s} w=w_{s s}+\frac{1}{s^{2}} w_{\phi \phi}+\frac{1}{s} w_{s}, \\
& D_{s} w=\left(s^{2} w_{s}\right)_{s} \\
& {[w, f]=\frac{1}{s}\left[w_{s s}\left(f_{s}+\frac{1}{s} f_{\phi \phi}\right)+f_{s s}\left(w_{s}+\frac{1}{s} w_{\phi \phi}\right)-\frac{2}{s}\left(f_{s \phi}-\frac{1}{s} f_{\phi}\right)\left(w_{s \phi}-\frac{1}{s} w_{\phi}\right)\right]} \\
& M(w, f)=-\left(D_{s} \Delta_{s}+\Delta_{s} D_{s}\right) f+\sin ^{2} \theta_{0} D_{s}^{2} f+2\left(\Delta_{s} f-\sin ^{2} \theta_{0} D_{s} f\right) \\
& +\tau\left(D_{s} w-2 w\right)+L_{1}(w, w) \text {, } \\
& N(w, f)=-\left(D_{s} \Delta_{s}+\Delta_{s} D_{s}\right) w+\sin ^{2} \theta_{0} D_{s}^{2} w+2\left(\Delta_{s} w-\sin ^{2} \theta_{0} D_{s} w\right) \\
& +\tau\left(-D_{s} f+2 f\right)+\lambda\left(-D_{s} w+2 w\right)-\left[L_{1}(w, f)+L_{1}(f, w)\right] \text {, } \\
& L_{1}(w, f)=-\left(s^{2} w_{s s}+s w_{s}\right)\left(\frac{1}{s^{2}} f_{\phi \phi}+\frac{1}{s} f_{s}-\left(\sin ^{2} \theta_{0}\right) s f_{s}\right) \\
& -s w_{s s} f_{s}-\left(w_{s \phi}-\frac{1}{s} w\right)\left(f_{s \phi}-\frac{1}{s} f_{\phi}\right) \\
& +w\left(\Delta_{s} f-\sin ^{2} \theta_{0} D_{s} f\right)+\sin ^{2} \theta_{0} f w, \\
& \tau=\sin \theta_{0} \frac{\rho_{0}}{h \gamma} \quad \text { and } \quad \lambda=\frac{\rho_{0}^{2} p}{E h^{3} \gamma^{2}} .
\end{aligned}
$$

Thus, $\tau$ is a measure of the initial curvature of the shell and $\lambda$ is a measure of the external load. 
Remark. In the derivation of (2.27)-(2.30), $\mathbf{r}=(\cos \phi \sin \theta, \sin \phi \sin \theta, \cos \theta)$ was used as the normal to $S_{R}(\Omega)$. This choice of orientation results in a nonnegative value of the parameter, $\tau$ (i.e., $\left.\tau=\left(\sin \theta_{0} \rho_{0}\right) /(h \gamma)\right)$. Clearly, if $(w, f, \lambda, \tau)$ with $\tau \geq 0$ is a solution of $(2.27)-(2.30)$, then $(-w, f, \lambda,-\tau)$ is also a solution. The solutions with $\tau<$ 0 correspond to the case when $-r$ is chosen as the unit normal to $S_{R}(\Omega)$. The results of choosing $-\mathrm{r}$ as a unit normal are that $\tilde{b}_{\alpha \beta}=(1 / R) \tilde{a}_{\alpha \beta}$ and the coefficients, $-1 / R$ and $1 / R$, appearing in (2.13) and (2.14) are replaced by $+1 / R$ and $-1 / R$, respectively. Repeating the previous analysis (with $-r$ as the unit normal), one is led to a system of equations identical to (2.27)-(2.30) with the exception that the parameter, $\tau$, will equal $-\left(\sin \theta_{0} \rho_{0}\right) /(h \gamma)$. Henceforth, we will consider both nonnegative and negative values of $\tau$.

Suppose $R$ is large and $\sin \theta_{0}$ is small. If $\rho_{0}=R \sin \theta_{0}$ is fixed, we can neglect terms of order $\sin ^{2} \theta_{0}$ in the above equations. Since $K=\sin ^{2} \theta_{0} / \rho_{0}^{2}, K$ is small (i.e., the cap is shallow) if and only if $\theta_{0}$ is small. Thus, in (2.27)-(2.30) we retain terms of order $\sin \theta_{0}$ and neglect terms of order $\sin ^{2} \theta_{0}$ to arrive at the shallow cap approximation to the John equations.

$$
\begin{gathered}
\Delta_{s}^{2} f+\frac{1}{2}[w, w]_{s}-\tau \Delta_{s} w=0 \quad \text { on } D, \\
\Delta_{s}^{2} w-[w, f]_{s}+\tau \Delta_{s} f+\lambda \Delta_{s} w=0 \text { on } D, \\
w=\Delta_{s} w=0 \text { on } \partial D, \\
f=\Delta_{s} f=0 \text { on } \partial D .
\end{gathered}
$$

A classical solution is a pair of functions, $w$ and $f$, that are four times continuously differentiable on $D$ (the unit disk), twice continuously differentiable on $\bar{D}$ and satisfy (2.31)-(2.34) pointwise.

Remark. If boundary conditions of the form (2.20)-(2.21) are imposed on equations (5) of Weinitschke [25, p. 143], one is also led to equations $(2.31)-(2.34)$.

If we let $\sin \theta_{0}$ approach 0 in (2.27)-(2.30), we obtain

$$
\begin{gathered}
\Delta_{s}^{2} f+\frac{1}{2}[w, w]_{s}=0 \quad \text { on } D, \\
\Delta_{s}^{2} w-[w, f]_{s}+\lambda \Delta_{s} w=0 \quad \text { on } D, \\
w=\Delta_{s} w=f=\Delta_{s} f=0 \quad \text { on } \partial D .
\end{gathered}
$$

Equations (2.35)-(2.37) are precisely the Von Kármán equations which describe the buckling of a circular plate. Thus, equations (2.31)-(2.34) occupy an intermediate position between the plate theory described by the Von Kármán equations and the shell theory of John, which makes no a priori assumptions on the initial curvature of the shell.

3. Hilbert space formulation. In the following, we shall drop the subscript " $s$ " on the operators $\Delta_{s}$ and [ , ] $]_{s}$. Instead of solving (2.31)-(2.34) directly, we shall first introduce an equivalent weak formulation (see concluding remarks for a discussion of this equivalence). Let $\Lambda$ be the set of smooth functions on $D$ which vanish on $\partial D$, where $D$ is the unit disk. Multiplying (2.31)-(2.32) by an arbitrary $\phi \in \Lambda$ and 
integrating by parts over $D$, we obtain

$$
\begin{gathered}
\int \Delta f \Delta \phi+\tau \int \nabla w \cdot \nabla \phi+\frac{1}{2} \int[w, w] \phi=0, \\
\int \Delta w \Delta \phi-\tau \int \nabla f \cdot \nabla \phi-\lambda \int \nabla w \cdot \nabla \phi-\int[w, f] \phi=0 .
\end{gathered}
$$

A weak (or generalized) solution is a pair of functions, $w$ and $f$, which satisfy (3.1)(3.2) for all $\phi \in \Lambda$. In the following, all integrals will be taken over $D$. We define

$$
\begin{aligned}
(f, \phi) & =\int \Delta f \Delta \phi, \\
a(w ; \phi) & =\int \nabla w \cdot \nabla \phi,
\end{aligned}
$$

and

$$
b(u, v ; \phi)=\int[u, v] \phi .
$$

Let $\mathscr{H}$ denote the Hilbert space obtained by completing $\Lambda$ relative to

$$
\|u\|^{2}=(u, u) \text {. }
$$

The norm, $\|u\|=(u, u)^{1 / 2}$, is equivalent to the usual Sobolev norm, $\|u\|_{2,2}$. Equations (3.3)-(3.5) define bounded linear functionals on $\mathscr{H}$. Hence, by the Riesz representation theorem, there exist elements in $\mathscr{H}$ such that

$$
\begin{aligned}
\int \Delta f \Delta \phi & =(f, \phi), \\
a(w ; \phi) & =(A w, \phi),
\end{aligned}
$$

and

$$
b(u, v ; \phi)=(B(u, v), \phi) .
$$

Thus, (3.1)-(3.2) have the equivalent operator formulation

$$
\begin{gathered}
f+\tau A w+\frac{1}{2} B(w, w)=0, \\
w-\tau A f-\lambda A w-B(w, f)=0 .
\end{gathered}
$$

Equation (3.10) can be used to eliminate $f$ in (3.11). This leads to

$$
L_{\lambda} w+\tau Q(w)+C(w)=0
$$

where

$$
\begin{aligned}
L_{\lambda} w & =w-\lambda A w+\tau^{2} A^{2} w \\
Q(w) & =\frac{1}{2} A B(w, w)+B(A w, w)
\end{aligned}
$$

and

$$
C(w)=\frac{1}{2} B(w, B(w, w)) .
$$


The derived operators, $Q^{\prime}$ and $C^{\prime}$, are given by

$$
\begin{aligned}
& Q^{\prime}(u) v=A B(u, v)+B(A u, v)+B(u, A v), \\
& C^{\prime}(u) v=B(u, B(u, v))+\frac{1}{2} B(v, B(u, u)) .
\end{aligned}
$$

The form $(B(u, v), w)$ for $u, v, w \in \mathscr{H}$ is symmetric, i.e., $(B(u, v), w)=(B(u, w), v)$ $=(B(v, w), u)$ (see [3]). The characteristic values of the operator $A$ are related to the eigenvalues of the following problem,

$$
\begin{aligned}
\Delta u+\mu_{0} u=0 & \text { on } D, \\
u=0 & \text { on } \partial D .
\end{aligned}
$$

LEMMA 1. $\mu_{0}$ is a characteristic value of $A$ if and only if $\mu_{0}$ is an eigenvalue of (3.17).

Proof. Suppose $\mu_{0} A u=u$. Standard regularity results imply that $u$ is smooth (see [1]). Let $\phi \in \Lambda$. Then

$$
\begin{aligned}
\mu_{0}(A u, \phi) & =(u, \phi), \\
\mu_{0} \int \nabla u \cdot \nabla \phi & =\int \Delta u \Delta \phi, \\
\int\left(\Delta^{2} u+\mu_{0} \Delta u\right) \phi & =0, \quad \text { for all } \phi \in \Lambda .
\end{aligned}
$$

Thus,

$$
\begin{array}{rlrl}
\Delta^{2} u+\mu_{0} \Delta u & =0 & & \text { on } D, \\
u=0 & & \text { on } \partial D,
\end{array}
$$

and

$$
\Delta u=0 \text { on } \partial D
$$

However, if $f \in \Lambda$, where

$$
\Delta f=0 \text { on } D,
$$

and

$$
f=0 \text { on } \partial D \text {, }
$$

then $f=0$ on $D$ by the maximum principle. Thus, from (3.18) we have

$$
\begin{aligned}
\Delta u+\mu_{0} u=0 & \text { on } D, \\
u=0 & \text { on } \partial D .
\end{aligned}
$$

Note, Eq. (3.18c) is satisfied by a solution of (3.17).

The eigenfunctions of (3.17) are given by

$$
u_{k}^{m}(s, \phi)=c_{k}^{m} J_{m}\left(j_{k}^{m} s\right) \cos m \phi, \quad m=0,1, \ldots, \quad k=1, \ldots,
$$

and 


$$
v_{k}^{m}(s, \phi)=d_{k}^{m} J_{m}\left(j_{k}^{m} s\right) \sin m \phi, \quad m=1,2, \ldots, \quad k=1, \ldots,
$$

where $J_{m}$ is a Bessel function of the first kind of order $m$. The corresponding eigenvalues are $\mu_{0}=\mu_{k}^{m}$, where $\mu_{k}^{m}=\left(j_{k}^{m}\right)^{2}$ and $J_{m}\left(j_{k}^{m}\right)=0$ for every integer $m \geq 0$ and $k=1,2, \ldots$ If $m$ and $p$ are integers with $m \geq 0$ and $p \geq 1, J_{m}$ and $J_{m+p}$ have no common zeroes other than the origin (see Watson [24, p. 484]). It follows that the eigenvalues, $\left\{\mu_{k}^{m}\right\}$, are distinct, $\mu_{k}^{0}$ has multiplicity one and $\mu_{k}^{m}(m \neq 0)$ has multiplicity two. The constants, $c_{k}^{m}$ and $d_{k}^{m}$, will be chosen such that

$$
\begin{aligned}
& \left(A u_{k}^{m}, u_{l}^{n}\right)=\delta_{n}^{m} \delta_{l}^{k}, \\
& \left(A v_{k}^{m}, v_{l}^{n}\right)=\delta_{n}^{m} \delta_{l}^{k} .
\end{aligned}
$$

Note, $\left(u_{k}^{m}, v_{l}^{n}\right)=0$ for all eigenfunctions. Using the fact that the eigenfunctions of (3.17) are complete in $L^{2}(D)$, one can show that the functions, $\left\{u_{k}^{0}, u_{k}^{m}, v_{k}^{m}\right\}$, are also complete in $\mathscr{H}$. Thus, $\mathscr{H}$ may be characterized as

$$
\mathscr{H}=\left\{u \mid u=\sum_{k=1}^{\infty}\left[a_{k}^{0} u_{k}^{0}+\sum_{m=1}^{\infty} a_{k}^{m} u_{k}^{m}+b_{k}^{m} v_{k}^{m}\right]\right\}
$$

Remark. In Section 2, we required that $\theta_{0}$ be sufficiently small in order to justify neglecting terms of order $\sin ^{2} \theta_{0}$. In addition, we shall require

$$
\tau^{2}=\frac{\sin ^{2} \theta_{0} \rho_{0}^{2}}{(h \gamma)^{2}}<\mu_{1}^{0} \mu_{1}^{1}
$$

We say $\lambda_{0}$ is an eigenvalue of $L_{\lambda}$ if $L_{\lambda_{0}} v=0$ for some $v \neq 0$. From the properties of the solutions of (3.17) and Lemma 1 , it follows that the characteristic values of $A$ have multiplicity one if $\mu_{0}=\mu_{k}^{0}$ and multiplicity two if $\mu_{0}=\mu_{k}^{m}$ for $m \neq 0$. The eigenvalues of $L_{\lambda}$ are related to the characteristic values of $A$ by the following lemma.

LemMA 2. $\left(\lambda_{0}, v\right)$ is an eigenpair for $L_{\lambda}$ if and only if $\lambda_{0}=\mu_{0}+\tau^{2} / \mu_{0}$ where $\mu_{0}$ is a characteristic value of $A$ with corresponding characteristic function, $v$.

Proof. The operator, $L_{\lambda_{0}}$, may be factored in the form,

$$
L_{\lambda_{0}} v=\left(I-\mu_{0} A\right)\left(I-\frac{\tau^{2}}{\mu_{0}} A\right) v=0 .
$$

The null space of $L_{\lambda_{0}}$ will be denoted by $\mathscr{N}$ where

$$
\mathscr{N}=\left\{u \mid L_{\lambda_{0}} u=0\right\} \text {. }
$$

If $\tau^{2}<\mu_{1}^{0} \mu_{1}^{1}$, then $\left(I-\left(\tau^{2} / \mu_{0}\right) A\right) v \neq 0$ for any $v \in \mathscr{H}$ (unless $\mu_{0}=|\tau|=\mu_{1}^{0}$, in which case both factors of $L_{\lambda_{0}}$ are $\left.\left(I-\mu_{0} A\right)\right)$ so that $\mathscr{N}$ is one-dimensional. Thus, the shallow cap assumption, (3.19), implies that the null spaces, $\mathscr{N}$, are one-dimensional in $\mathscr{H}$ when $\mu_{0}=\mu_{k}^{0}\left(\mathscr{N}=\left[u_{k}^{0}\right]\right)$, and two-dimensional in $\mathscr{H}$ when $\mu_{0}=\mu_{k}^{m}\left(\mathscr{N}=\left[u_{k}^{m}, v_{k}^{m}\right]\right)$. Here, $\left[u_{1}, \ldots, u_{n}\right]$ denotes the subspace spanned by $u_{1}, \ldots, u_{n}$.

4. Group invariance. In this section, we will show that every nonaxisymmetric solution of $(*)$ generates a one-parameter family of solutions. We will also discuss how this affects the methods of solving $(*)$ that are based on the usual implicit function theorem. This situation is most conveniently described by using group representations on the Hilbert space, $\mathscr{H}$. 
Let $g \rightarrow T_{g}$ be a representation of the group, $\mathscr{O}(2)$, on $\mathscr{H}$ given by

$$
T_{g} w=w\left(g^{-1} x\right) \quad \text { for } g \in \mathscr{O}(2), x \in D, w \in \mathscr{H} \text {. }
$$

By using the property that $\Delta T_{g} w=T_{g} \Delta w$ for $g \in \mathscr{O}(2)$ and $w \in \Lambda$ (see [18, p. 65]), it can be shown that $g \rightarrow T_{g}$ is unitary with respect to the inner product, $(u, v)=\int_{D} \Delta u \Delta v$ (i.e., $\left.\left(T_{g} u, T_{g} v\right)=(u, v)\right)$. Furthermore, using a result of Knightly and Sather, [13, Lemma 4], the operators, $A, Q$, and $C$, are invariant under $\mathscr{O}(2)$ in the sense that $T_{g} A w=A\left(T_{g} w\right), T_{g} Q(w)=Q\left(T_{g} w\right)$, and $T_{g} C(w)=C\left(T_{g} w\right)$. It follows that

$$
\begin{aligned}
0 & =T_{g}\left(L_{\lambda} w+\tau Q(w)+C(w)\right) \\
& =L_{\lambda}\left(T_{g} w\right)+\tau Q\left(T_{g} w\right)+C\left(T_{g} w\right) .
\end{aligned}
$$

Thus, if $w$ is a solution of $(*)$, then $T_{g} w$ is also a solution of $(*)$ for all $g \in \mathscr{O}(2)$. If $w$ is an axisymmetric solution, then $T_{g} w=w$ for all $g \in \mathscr{O}(2)$. However, when $w$ is nonaxisymmetric, the consequences of the above remark are as follows:

1) A nonaxisymmetric solution of $(*)$ generates a one-parameter family of solutions. If $g \in \mathscr{O}(2)$ corresponds to a rotation of $\alpha$ degrees about the $z$-axis, then $T_{g} w=w(s, \phi-\alpha)$. If $w$ solves $(*)$, then every element of $\mathscr{M}=\left\{w_{\alpha} \mid w_{\alpha}=w(s, \phi-\alpha)\right\}$ also solves $(*)$.

2) Solutions of $(*)$ are not isolated. In every neighborhood of a solution, $w$, there exist other solutions of the form, $w_{\alpha}=w(s, \phi-\alpha)$, with $\alpha$ arbitrarily small and $\left\|w_{\alpha}-w\right\| \neq 0$.

3) The usual arguments based on the implicit function theorem cannot be applied directly. Suppose $w^{*}$ solves $F(w)=0$, where $F(w)=L_{\lambda} w+\tau Q(w)+C(w)$. By (1), $w_{\alpha}=w^{*}(s, \phi-\alpha)$ is also a solution for every $\alpha$. Thus, $0=F\left(w_{\alpha}\right)-F\left(w^{*}\right)=$ $F^{\prime}\left(w^{*}\right) \alpha+r\left(w^{*}, \alpha\right)$, where $\|r\|=o(\alpha)$ (i.e., $\left.\lim _{\alpha \rightarrow 0}\|r\| / \alpha=0\right)$. Hence, $F^{\prime}\left(w^{*}\right)$ is not invertible for such a solution of $(*)$. Consequently, the usual implicit function theorem cannot be used to solve $F(w)=0$ in a neighborhood of the known solution, $w^{*}$.

One could avoid these problems by assuming the solutions are axisymmetric as in [12] and [20]. An alternate approach is to require the solutions of $(*)$ to have a minimal degree of symmetry. For example, one could require the following. If $w(s, \phi)$ is a solution, there exists a plane, $\phi=t^{*}$, about which $w$ is symmetric; in other words, there is a $t^{*}$ such that for every $0 \leq s \leq 1$ and $0 \leq \phi \leq 2 \pi$,

$$
w\left(s, t^{*}-\phi\right)=w\left(s, t^{*}+\phi\right) .
$$

If we require solutions to possess this reflectional symmetry, a unique element in $\mathscr{M}$ will be determined. The solutions in this sense will be isolated for fixed $\lambda$ and $\tau$.

Remark. The experimental evidence in [5] and [6] suggests that the observed solutions do possess the reflectional symmetry discussed above. The least symmetric buckled state observed is that of a pear-shaped dimple, which possesses one axis of reflectional symmetry. However, it is still an open question as to whether or not the above procedure will yield all the solutions of $(*)$ branching from the trivial solution. 
Condition (P1) actually implies that one need only consider solutions of the form

$$
w(s, \phi)=\sum_{m=0}^{\infty} \sum_{k=1}^{\infty} a_{k}^{m} u_{k}^{m},
$$

as shown by the following result.

Lemma 3. If $w \in \mathscr{H}$ satisfies $w\left(s, t^{*}-\phi\right)=w\left(s, t^{*}+\phi\right)$ for some $t^{*}$, then $w$ can be written in the form

$$
w=\sum_{k=1}^{\infty}\left\{a_{k}^{0} u_{k}^{0}+\sum_{m=1}^{\infty} \frac{a_{k}^{m}}{\cos m t^{*}} J_{m}\left(j_{k}^{m} s\right) \cos \left(m\left(\phi-t^{*}\right)\right)\right\} .
$$

Proof. $w \in \mathscr{H}$ implies $w(s, \phi)=\sum_{k=1}^{\infty}\left\{a_{k}^{0} u_{k}^{0}+\sum_{m=1}^{\infty} a_{k}^{m} u_{k}^{m}+b_{k}^{m} v_{k}^{m}\right\}$. Hence,

$$
w(s, \phi+t)=\sum_{k=1}^{\infty}\left\{a_{k}^{0} u_{k}^{0}+\sum_{m=1}^{\infty} J_{m}\left(j_{k}^{m} s\right)\left[a_{k}^{m} \cos (m(\phi+t))+b_{k}^{m} \sin (m(\phi+t))\right]\right\} \text {. }
$$

Using the fact that

$$
\begin{aligned}
a_{k}^{m} \cos (m(\phi+t))+b_{k}^{m} \sin (m(\phi+t))=\left(a_{k}^{m} \cos m t+b_{k}^{m} \sin m t\right) \cos m \phi \\
+\left(-a_{k}^{m} \sin m t+b_{k}^{m} \cos m t\right) \sin m \phi,
\end{aligned}
$$

and the condition, $w\left(s, t^{*}-\phi\right)=w\left(s, t^{*}+\phi\right)$, we obtain

$$
b_{k}^{m} \cos m t^{*}=a_{k}^{m} \sin m t^{*} \quad \text { for } m=1,2, \ldots, \quad k=1,2, \ldots
$$

Since $\cos m t^{*} \neq 0$ for all $m=1,2, \ldots$, it follows that

$$
w(s, \phi)=\sum_{k=1}^{\infty}\left\{a_{k}^{0} u_{k}^{0}+\sum_{m=1}^{\infty} \frac{J_{m}\left(j_{k}^{m} s\right)}{\cos m t^{*}}\left[a_{k}^{m} \cos \left(m t^{*}\right) \cos (m \phi)+b_{k}^{m} \cos \left(m t^{*}\right) \sin (m \phi)\right]\right\} .
$$

Substituting (4.4) into (4.5) and simplifying gives the desired result.

Note, if $\cos \left(m t^{*}\right)=0$ for some $m$, then $a_{k}^{m}=0$, since $\sin \left(m t^{*}\right) \neq 0$. Hence (4.2) holds true with the convention that $a_{k}^{m} / \cos \left(m t^{*}\right)=0$ whenever $a_{k}^{m}=0$.

If one is interested in solutions of $(*)$ that satisfy the symmetry assumption, (P1), Lemma 3 implies one need only consider solutions that are even about $\phi=t^{*}$. For our convenience, we choose $t^{*}=0$. Hence, the solutions satisfying (P1) will lie in the subspace, $\mathscr{W}$, where

$$
\mathscr{W}=\left\{w \mid w \in \mathscr{H}, w=\sum_{m=0}^{\infty} \sum_{k=1}^{\infty} a_{k}^{m} u_{k}^{m}\right\} .
$$

Representations of the group, $\mathscr{O}(2)$, can then be applied to each nonaxisymmetric solution in $\mathscr{W}$ to generate the solutions in $\mathscr{H}$ which are not even about $\phi=0$.

Remark. The operators, $Q$ and $C$, keep the subspace $\mathscr{W}$ invariant. This is seen by inspecting $[u, v]$ and the definitions of $Q$ and $C$. 
5. Determination of bifurcating solutions. We next outline the Liapunov-Schmidt procedure for solving

$$
w-\lambda A w+\tau^{2} A^{2} w+\tau Q(w)+C(w)=0, \quad \text { for } w \in \mathscr{W} .
$$

Let

$$
\begin{aligned}
w & =\tau(u+U) \quad \text { where } u \in \mathscr{N}, U \in \mathcal{N}^{\perp}, \mathscr{W}=\mathscr{N} \oplus \mathcal{N}^{\perp}, \\
\lambda & =\mu_{0}+\frac{\tau^{2}}{\mu_{0}}+\tau^{2} \mu_{0} t, \\
\mu_{0} & =\mu_{k}^{m} \quad \text { for some } m=0,1, \ldots, \quad k=1,2, \ldots, \\
\mathscr{N} & =\left\{u \mid L_{\lambda_{0}} u=0\right\} \quad \text { where } \lambda_{0}=\mu_{0}+\tau^{2} / \mu_{0}, \text { and }
\end{aligned}
$$

$\mathscr{N}^{\perp}$ is the orthogonal complement of $\mathscr{N}$. Substituting (5.1) and (5.2) into (*) we obtain

$$
\tau L_{\lambda_{0}}(u+U)-\mu_{0} \tau^{3} t A(u+U)+\tau^{3} Q(u+U)+\tau^{3} C(u+U)=0 .
$$

Let $S$ denote the orthogonal projection of $\mathscr{W}$ onto $\mathscr{N}$ and let $I-S$ denote the orthogonal projection of $\mathscr{W}$ onto $\mathscr{N}^{\perp}$. Projecting (5.3) onto $\mathscr{N}$ and $\mathscr{N}^{\perp}$, we obtain

$$
\begin{gathered}
-\mu_{0} t A u+S[Q(u)+C(u)]+s=0, \\
L_{\lambda_{0}} U+\tau^{2}(I-S)\{-t A U+Q(u+U)+C(u+U)\}=0,
\end{gathered}
$$

where

$$
s=s(u, U)=S\left\{Q^{\prime}(u) U+Q(U)+C^{\prime}(u) U+C^{\prime}(U) u+C(U)\right\} .
$$

Since the null spaces, $\mathcal{N}$, are all one-dimensional in $\mathscr{W}$, Equation (5.4) may be rewritten in the form

$$
0=F(t, \beta, \tau)
$$

where

$$
F(t, \beta, \tau)=-t \beta+(Q(z), z) \beta^{2}+(C(z), z) \beta^{3}+(s, z),
$$

$u=\beta z, \beta \in \mathbf{R}^{1},(A z, z)=1$, and $z=u_{k}^{m}$.

Equation (5.5) can be solved for $U$ in the following manner. Given positive numbers $\rho_{1}, R, t_{1}$, there exists a positive number $\tau_{1}$, such that for $|\beta|<\rho_{1},|t|<t_{1}$, and $|\tau|<\tau_{1}$, equation (5.5) defines a contraction on $\|U\|<R$ and can be solved for $U=U(t, \beta, \tau) \in \mathscr{N}^{\perp}$ by the contraction mapping principle. The solution, $U=U(t, \beta, \tau)$, is analytic in $(t, \beta, \tau)$ by the uniform contraction principle (see [7, p. 25]) and satisfies

$$
\|U(t, \beta, \tau)\|<k_{1} \beta^{2} \tau^{2} \quad \text { for }|t|<t_{1},|\beta|<\rho_{1} \text {, and }|\tau|<\tau_{1} .
$$

It follows from (5.6) that if $U$ is a solution of (5.5) obtained in this way, then

$$
\|s\|=\|s(\beta z, U(t, \beta, \tau))\| \leq k_{2} \beta^{3} \tau^{2} \text { for }|t|<t_{1},|\beta|<\rho_{1},|\tau|<\tau_{1} .
$$

Moreover, substituting the solution, $U=U(t, \beta, \tau)$, into (5.7), we see that (5.7) can be written in the form,

$$
0=F(t, \beta, \tau)=\beta G(t, \beta, \tau),
$$

where

$$
G(t, \beta, \tau)=-t+q \beta+c \beta^{2}+r(t, \beta, \tau),
$$


$q=(Q(z), z), c=(C(z), z)$, and $r(t, \beta, \tau)=\beta^{-1}(s, z)$. From (5.10) it follows that

$$
|r(t, \beta, \tau)| \leq k_{3} \beta^{2} \tau^{2} \quad \text { for }|t|<t_{1},|\beta|<\rho_{1} \text {, and }|\tau|<\tau_{1} .
$$

By the definition of $G$ in (5.12a) and the analyticity of $U$, it follows that $G$ is also analytic on $\mathscr{G}=\left(-t_{1}, t_{1}\right) \times\left(-\rho_{1}, \rho_{1}\right) \times\left(-\tau_{1}, \tau_{1}\right)$.

One obvious solution of $(5.11)$ is $\beta=0$. In what follows, we shall consider those solutions of (5.11) for which

$$
0=G(t, \beta, \tau)
$$

The solutions of (5.13) are closely related to the solutions of the reduced branching equation,

$$
0=G(t, \beta, 0)=-t+q \beta+c \beta^{2} .
$$

Equation (5.14) is obtained by letting $\tau \rightarrow 0$ in Equation (5.13) and applying (5.12). In the following, let $C_{0}=\left\{(t, \beta, 0) \mid t=q \beta+c \beta^{2}\right\} \cap \tilde{\mathscr{G}}$ where $\tilde{\mathscr{G}}=\left[-\tilde{t}_{1}, \tilde{t}_{1}\right] \times\left[-\tilde{\rho}_{1}, \tilde{\rho}_{1}\right] \times$ $\left[-\tilde{\tau}_{1}, \tilde{\tau}_{1}\right], \tilde{t}_{1}<t_{1}, \tilde{\rho}_{1}<\rho_{1}$, and $\tilde{\tau}_{1}<\tau_{1}$. If $\left(t_{k}^{*}, \beta_{k}^{*}, 0\right) \in C_{0}$, it follows that

$$
G_{t}\left(t^{*}, \beta^{*}, 0\right)=-1 \text {. }
$$

Since $G$ is analytic on $\tilde{\mathscr{G}},\left(t_{k}^{*}, \beta_{k}^{*}, 0\right) \in C_{0}$, and $G_{t}\left(t^{*}, \beta^{*}, 0\right) \neq 0$, by the implicit function theorem we have the following. There exist sets, $\mathscr{T}_{k}=\left\{t \mid \alpha_{k}<t-t_{k}^{*}<\alpha_{k}^{\prime}\right\}$, $\mathscr{U}_{k}=\left\{\beta \mid \varepsilon_{k}<\beta-\beta_{k}^{*}<\varepsilon_{k}^{\prime}\right\}, \mathscr{V}_{k}=\left\{\tau \mid-\delta_{k}<\tau<\delta_{k}\right\}$ and a function, $t_{k}(\beta, \tau)$, such that

(i) $\mathscr{T}_{k} \times \mathscr{U}_{k} \times \mathscr{V}_{k} \subset \tilde{\mathscr{G}}$,

(ii) $t_{k}(\beta, \tau)$ is unique and analytic on $\mathscr{U}_{k} \times \mathscr{V}_{k}\left(t_{k} \mid \mathscr{U}_{k} \times \mathscr{V}_{k} \rightarrow \mathscr{T}_{k}\right)$,

(iii) $\lim _{(\beta, \tau) \rightarrow\left(\beta_{k}^{*}, 0\right)} t_{k}(\beta, \tau)=t_{k}^{*}$, and

(iv) $G\left(t_{k}(\beta, \tau), \beta, \tau\right)=0$ for $(\beta, \tau) \in \mathscr{U}_{k} \times \mathscr{V}_{k}$.

Since $C_{0}$ is compact, we can choose a finite set of points, $\left\{\left(t_{k}^{*}, \beta_{k}^{*}, 0\right)\right\}_{k=1}^{N}$, such that $\left(t_{k}^{*}, \beta_{k}^{*}, 0\right) \in C_{0}$ and the corresponding sets, $\mathscr{T}_{k} \times \mathscr{U}_{k} \times \mathscr{V}_{k}$, cover $C_{0}$ (i.e., $C_{0} \subset$ $\left.\bigcup_{k=1}^{N}\left(\mathscr{T}_{k} \times \mathscr{U}_{k} \times \mathscr{V}_{k}\right)\right)$. By the local uniqueness of the $t_{k}(\beta, \tau)$ 's, we have $t_{k}(\beta, \tau)=$ $t_{j}(\beta, \tau)$ for $(\beta, \tau) \in \mathscr{U}_{k} \times \mathscr{V}_{k} \cap \mathscr{U}_{j} \times \mathscr{V}_{j}$. Henceforth, we can omit the subscript, $k$, on the $t_{k}(\beta, \tau)$ 's. Furthermore, by (5.12) we have $\left|t(\beta, \tau)-q \beta-c \beta^{2}\right|<K \tau$ for $(\beta, \tau) \in \bigcup_{k=1}^{N}\left(\mathscr{U}_{k} \times \mathscr{V}_{k}\right), K=k_{3} \rho_{1}^{2} \tau_{1}$. Let $\tau_{0}$ be a positive number satisfying $\tau_{0}<$ $\min \left\{\tilde{\tau}_{1},\left|\alpha_{k}\right| / K,\left|\alpha_{k}^{\prime}\right| / K, \delta_{k}\right.$, for $\left.k=1, \ldots, N\right\}$. With no loss of generality, we can choose $\rho_{0}$ such that $(t(\beta, \tau), \beta, \tau)$ is a solution of $(5.13)$ for $|\beta|<\rho_{0}$ and $|\tau|<\tau_{0}$. Note, $\rho_{0}$ is not necessarily small; it is determined by the set on which $r(t, \beta, \tau)$ is analytic. For fixed $\tau^{\prime},\left(t\left(\beta, \tau^{\prime}\right), \beta\right)$ describes a "perturbed parabola" in the $\tau=\tau^{\prime}$ plane. We are led to the following.

THEOREM 1. Let $\mu_{k}^{m}$ be a characteristic value of $A$ with corresponding characteristic function, $u_{k}^{m}$. There exist positive contants, $\rho_{0}$ and $\tau_{0}$, and analytic functions, $t(\beta, \tau)$ and $U(t(\beta, \tau), \beta, \tau)$, such that

$$
\begin{aligned}
(w, \lambda) & =(w(\beta, \tau), \lambda(\beta, \tau)) \\
& =\left(\tau\left(\beta u_{k}^{m}+U(t(\beta, \tau), \beta, \tau)\right), \mu_{k}^{m}+\tau^{2}\left(1 / \mu_{k}^{m}+\mu_{k}^{m} t(\beta, \tau)\right)\right)
\end{aligned}
$$

is a solution of $(*)$ for all $-\rho_{0}<\beta<\rho_{0}$ and $-\tau_{0}<\tau<\tau_{0}$.

The form of $\lambda(\beta, \tau)$ follows by substituting the function, $t=t(\beta, \tau)$, into (5.2). 
Before proceeding to the specific bifurcation equations, we note the following results.

LemMA 4. Let $\left\{u_{k}^{0}, u_{k}^{m}, v_{k}^{m}\right\}$ denote the characteristic functions of $A$.

(i) If $u \in \mathscr{N}=\left[u_{k}^{m}, v_{k}^{m}\right]$ for $m \neq 0$, then $S Q(u)=0$.

(ii) $q=\left(Q\left(u_{k}^{0}\right), u_{k}^{0}\right)>0$.

(iii) If $z \in \mathscr{H}$ and $z \neq 0$, then $c=(C(z), z)=\frac{1}{2}\|B(z, z)\|^{2}>0$.

Proof of (i). Let

$$
\begin{aligned}
& z_{1}=u_{k}^{m}=c_{k}^{m} J_{m}\left(j_{k}^{m} s\right) \cos m \phi, \\
& z_{2}=v_{k}^{m}=d_{k}^{m} J_{m}\left(j_{k}^{m} s\right) \sin m \phi .
\end{aligned}
$$

By integrating out the " $\phi$ " variable in each integral of the form

$$
\int_{0}^{2 \pi} \int_{0}^{1}\left[z_{i}, z_{j}\right] z_{k} d A, \quad \text { where } d A=s d s d \phi, \quad i, j, k \in\{1,2\},
$$

and noting

$$
\begin{aligned}
0=\int_{0}^{2 \pi} \sin ^{3} m \phi d \phi=\int_{0}^{2 \pi} \cos ^{3} m \phi d \phi & =\int_{0}^{2 \pi} \cos ^{2} m \phi \sin m \phi d \phi \\
& =\int_{0}^{2 \pi} \sin ^{2} m \phi \cos m \phi d \phi,
\end{aligned}
$$

we obtain the following:

$$
\left(B\left(z_{i}, z_{j}\right), z_{k}\right)=\int_{D}\left[z_{i}, z_{j}\right] z_{k} d A=0 \text { for } i, j, k \in\{1,2\} .
$$

Thus,

$$
\left(Q\left(z_{i}\right), z_{j}\right)=\frac{3}{2 \mu_{k}^{m}}\left(B\left(z_{i}, z_{i}\right), z_{j}\right)=0 \text { for } i, j \in\{1,2\} .
$$

Statement (i) of the lemma follows by applying (3.13) and (5.17) to $S Q\left(\xi_{1} z_{1}+\xi_{2} z_{2}\right)$ for $\xi_{1}, \xi_{2} \in \mathbf{R}^{1}$.

Proof of (ii). Let $z=u_{k}^{0}=J_{0}\left(j_{k}^{0} s\right)$; it follows that

$$
[z, z]=\frac{2}{s} z_{s s} z_{s}=\frac{1}{s}\left[\left(z_{s}\right)^{2}\right]_{s}
$$

Thus,

$$
b(z, z ; z)=2 \pi \int_{0}^{1}\left[\left(z_{s}\right)^{2}\right]_{s} z d s .
$$

Integrating the above expression by parts, we obtain

$$
b(z, z ; z)=2 \pi\left[\left.z\left(z_{s}\right)^{2}\right|_{0} ^{1}-\int_{0}^{1}\left(z_{s}\right)^{3} d s\right] .
$$

Note, $z(1)=J_{0}\left(j_{k}^{0}\right)=0$. Since $\frac{d}{d x} J_{0}(x)=-J_{1}(x)$ and $J_{1}(0)=0$, we have $z_{s}(0)=0$. Thus,

$$
b(z, z ; z)=-2 \pi\left(c_{k}^{0}\right)^{3} \int_{0}^{1}\left[\frac{d}{d s} J_{0}\left(j_{k}^{0} s\right)\right]^{3} d s .
$$




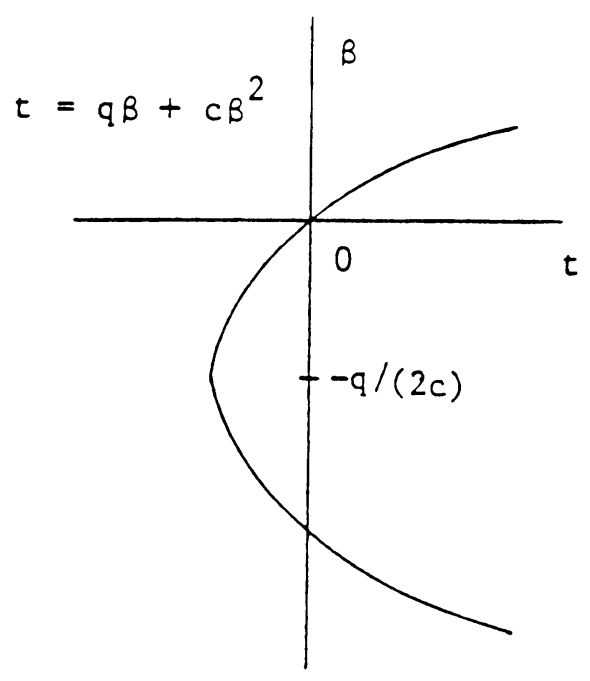

FIG. 5.1a. Bifurcation from a simple eigenvalue for a shallow cap in the $\tau=0$ plane.

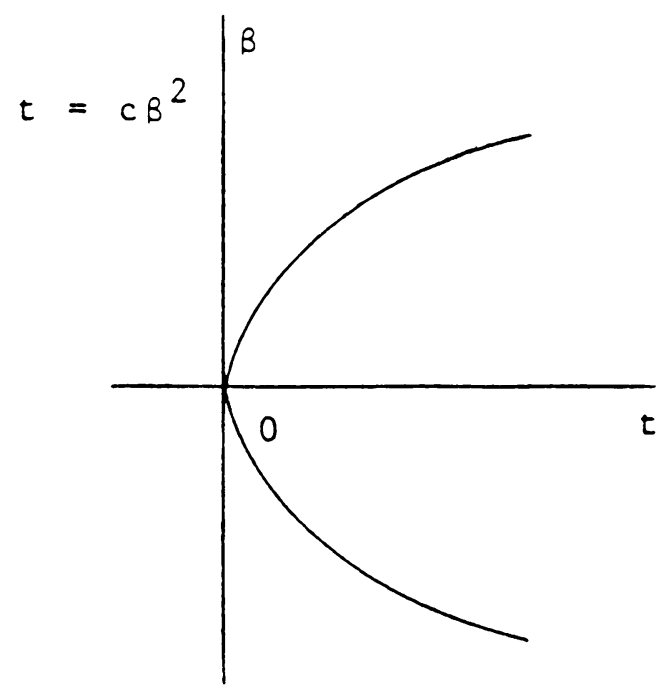

FIG. 5.1b. Bifurcation from a double eigenvalue for a shallow cap in the $\tau=0$ plane.

If we let $x=j_{k}^{0} s$, we obtain

Hence,

$$
b(z, z ; z)=-2 \pi\left(j_{k}^{0}\right)^{2}\left(c_{k}^{0}\right)^{3} \int_{0}^{j_{k}^{0}}\left[\frac{d}{d x} J_{0}(x)\right]^{3} d x
$$

$$
b(z, z ; z)=2 \pi\left(j_{k}^{0}\right)^{2}\left(c_{k}^{0}\right)^{3} \int_{0}^{j_{k}^{0}}\left[J_{1}(x)\right]^{3} d x>0 .
$$

The inequality, (5.18), follows from a result by Knightly and Sather [12]. Since $(Q(z), z)=3\left(2 \mu_{k}^{0}\right)^{-1} b(z, z ; z)$, (ii) is proven.

Proof of (iii). Let $c=\frac{1}{2}\|B(z, z)\|^{2}$ and $z \in \mathscr{H}$. Since $B(z, z)=0$ if and only if $z=0$ (see [8, p. 85]), we have $c>0$.

Suppose $\lambda_{0}=\mu_{0}+\tau^{2} / \mu_{0}$ is an eigenvalue of $L_{\lambda}$. From Lemma 4 and (5.14) the reduced branching equations are given by

and

$$
0=-t+q \beta+c \beta^{2}, \quad \text { if } \mu_{0}=\mu_{k}^{0},
$$

$$
0=-t+c \beta^{2}, \quad \text { if } \mu_{0}=\mu_{k}^{m} \text { and } \quad m \neq 0 .
$$

The solutions of (5.19) and (5.20) are sketched in Figures 5.1a and 5.1b, respectively.

From (5.19) and (5.20), we conclude the following. If $\lambda_{0}$ is simple in $\mathscr{H}$, then solutions bifurcate transcritically from the trivial solution. If $\lambda_{0}$ has multiplicity two in $\mathscr{H}$, then the corresponding solutions bifurcate supercritically. For shallow caps, we conclude there are no subcritical nonaxisymmetric solutions near a branch point.

6. Stability. Stability will be defined as in [21] or [22]. A solution of $\mathscr{F}(w, \lambda)=0$ is said to be stable if all the eigenvalues of the derived operator are positive, unstable if at least one eigenvalue is negative, and indeterminate otherwise. If

$$
\mathscr{F}(w, \lambda)=L_{\lambda} w+\tau Q(w)+C(w),
$$


then

$$
\begin{aligned}
\mathscr{F}^{\prime}(w, \lambda) h & =\left.\frac{d}{d \sigma} \mathscr{F}(w+\sigma h, \lambda)\right|_{\sigma=0}, \\
& =\left(I-\lambda A+\tau^{2} A^{2}\right) h+\tau Q^{\prime}(w) h+C^{\prime}(w) h .
\end{aligned}
$$

Let $w^{*}=\tau\left(\beta^{*} z+U\left(t^{*}, \beta^{*}, \tau\right)\right)$ be a solution of $(*)$ and

$$
\lambda^{*}=\mu_{1}+\frac{\tau^{2}}{\mu_{1}}+\mu_{1} \tau^{2} t^{*} \quad \text { with } \mu_{1}=\mu_{k}^{m} \text { and } z=u_{k}^{m} .
$$

We define $u^{*}=\beta^{*} z$. The derived operator, evaluated at $\left(w^{*}, \lambda^{*}\right)$, is given by

$$
\begin{aligned}
\mathscr{F}^{\prime}\left(w^{*}, \lambda^{*}\right) & =I-\lambda^{*} A+\tau^{2} A^{2}+\tau Q^{\prime}\left(w^{*}\right)+C^{\prime}\left(w^{*}\right) \\
& =\left(I-\mu_{1} A\right)+\tau^{2}\left[A^{2}-\frac{1}{\mu_{1}} A-\mu_{1} t^{*} A+Q^{\prime}\left(u^{*}\right)+C^{\prime}\left(u^{*}\right)\right]+N(\tau),
\end{aligned}
$$

where

$$
\|N(\tau)\|=o\left(\tau^{2}\right)
$$

The operator, $\mathscr{F}^{\prime}\left(w^{*}, \lambda^{*}\right)$, is a symmetric perturbation of a self-adjoint operator (see $[14$, p. 226]) and is therefore itself a self-adjoint operator with only real eigenvalues (see Kato, [11, p. 287]). If $\mu_{k}^{m} \neq \mu_{1}^{0}$, then $I-\mu_{k}^{m} A$ has a negative eigenvalue. It follows that $\mathscr{F}^{\prime}$ also has a negative eigenvalue (for small $\tau$ ) and the solution, $w^{*}$, is unstable. Thus, for a shallow cap, stable solutions (of small norm) must bifurcate from a critical eigenvalue of the form

$$
\lambda_{c}=\mu_{1}^{0}+\tau^{2} / \mu_{1}^{0} .
$$

Let $(w, \lambda)$ be a solution of $(*)$ (as found in Section 5), where

$$
\begin{aligned}
w(\beta, \tau) & =\tau(\beta z+U(t(\beta, \tau), \beta, \tau)) \text { for }-\rho_{0}<\beta<\rho_{0} \text { and }-\tau_{0}<\tau<\tau_{0}, \\
\lambda & =\mu_{1}+\tau^{2} / \mu_{1}+\mu_{1} t(\beta, \tau) \tau^{2}, \\
\mu_{1} & =\mu_{1}^{0}, \\
z & =u_{1}^{0} .
\end{aligned}
$$

From (5.12a) and (5.13), it follows that $t(\beta, \tau)$ can be expressed in the form

$$
t(\beta, \tau)=q \beta+c \beta^{2}+r(t(\beta, \tau), \beta, \tau) \text { for }-\rho_{0}<\beta<\rho_{0} \text { and }-\tau_{0}<\tau<\tau_{0} .
$$

After substituting (6.7)-(6.9) into (6.4), we see that the derived operator of $\mathscr{F}$, evaluated at $(w, \lambda)$, can be expressed in the form

$$
\begin{aligned}
D(\beta, \tau) & =\mathscr{F}^{\prime}(w, \lambda) \\
& =\left(I-\mu_{1} A\right)-\left(\tau^{2} / \mu_{1}\right) A\left(I-\mu_{1} A\right)+\tau^{2} M(\beta)+R(\tau),
\end{aligned}
$$

where

$$
\begin{aligned}
M(\beta) & =-\mu_{1}\left(q \beta+c \beta^{2}\right) A+\beta Q^{\prime}(z)+\beta^{2} C^{\prime}(z), \\
\|R(\tau)\| & =o\left(\tau^{2}\right) .
\end{aligned}
$$

In order to determine the stability of $w(\beta, \tau)$, we will use an implicit function theorem argument of McLeod and Sattinger [17] to construct the unique one-dimensional invariant subspace, $[\phi]$, in the form

$$
\phi=z+\Psi(\beta, \tau)
$$


for which

$$
D(\beta, \tau) \phi-\left(\tau^{2} / \mu_{1}\right) b(\beta, \tau) \phi=0,
$$

for $-\tilde{\rho}_{0}<\beta<\tilde{\rho}_{0}$ and $-\tilde{\tau}_{0}<\tau<\tilde{\tau}_{0}$ and $\Psi(\beta, \tau) \in \mathcal{N}^{\perp}$. The principle eigenvalue of $D(\beta, \tau)$ is simple for $|\beta|+|\tau|$ sufficiently small (see [11]). Thus, $\tau^{2} \mu_{1}^{-1} b(\beta, \tau)$ correctly characterizes this eigenvalue. The constants $\tilde{\rho}_{0}$ and $\tilde{\tau}_{0}$ will be defined later. Here, we present the necessary steps required to construct $[\phi]$. First, projecting (6.12) onto $\mathcal{N}^{\perp}$ we obtain

$$
\begin{aligned}
0= & F_{1}(\Psi, b, \beta, \tau) \\
= & \left(I-\mu_{1} A\right) \Psi-\left(\tau^{2} / \mu_{1}\right) A\left(I-\mu_{1} A\right) \Psi+\tau^{2}(I-S) M(\beta) \phi \\
& +(I-S) R(\tau) \phi-\left(\tau^{2} / \mu_{1}\right) b(\beta, \tau) \Psi .
\end{aligned}
$$

Projecting (6.12) onto $\mathcal{N}$ and dividing the result by $\tau^{2}$, we obtain

$$
\begin{aligned}
0 & =F_{2}(\Psi, b, \beta, \tau) \\
& =S M(\beta)(z+\Psi)+\tau^{-2} S R(\tau)(z+\Psi)-\mu_{1}^{-1} b(\beta, \tau) z
\end{aligned}
$$

Equations (6.13)-(6.14) define a map, $\mathbf{F}=\left(F_{1}, F_{2}\right)$, where $\mathbf{F}: \mathscr{N}^{\perp} \times \mathbf{R}^{3} \rightarrow \mathscr{N}^{\perp} \times \mathscr{N}$. The problem of determining $[\phi]$ is now reduced to solving

$$
\mathbf{0}=\mathbf{F}(\Psi, b, \beta, \tau) \text {. }
$$

By inspection, a nonzero solution of $(6.15)$ at $\tau=0$ is given by $(\Psi, b, \beta, \tau)=$ $\left(0, b^{*}, \beta^{*}, 0\right)$ where $\left(\beta^{*}, 0\right) \in\left(-\rho_{0}, \rho_{0}\right) \times\left(-\tau_{0}, \tau_{0}\right)\left(\rho_{0}\right.$ and $\tau_{0}$ are defined in Section 5) and $b^{*}=b\left(\beta^{*}, 0\right)=q \beta^{*}+2 c \beta^{*^{2}}$. Given positive constants, $R$ and $b_{0}\left(\left|b^{*}\right|<b_{0}\right)$, (6.15) can be solved in a neighborhood of $\left(\beta^{*}, 0\right)$ for $\Psi(\beta, \tau)$ and $b(\beta, \tau)$ such that $\|\Psi\|<R$ and $|b|<b_{0}$, in the following manner. The Frechet derivative of $\mathbf{F}$ with respect to $\Psi$ and $b$, evaluated at $(\Psi, b, \beta, \tau)=\left(0, b^{*}, \beta^{*}, 0\right)$, is given by

$$
\mathbf{F}^{\prime}\left(0, b^{*}, \beta^{*}, 0\right)=\left[\begin{array}{cc}
I-\mu_{1} A & 0 \\
S M\left(\beta^{*}\right) & -\mu_{1}^{-1} I
\end{array}\right],
$$

which is invertible from $\mathscr{N}^{\perp} \times \mathscr{N} \rightarrow \mathscr{N}^{\perp} \times \mathscr{N}$. Suppose $\mathbf{F}^{\prime}\left(0, b^{*}, \beta^{*}, 0\right)$ was not invertible. Then 0 would be an eigenvalue of $\mathbf{F}^{\prime}\left(0, b^{*}, \beta^{*}, 0\right)$ and there would exist $H \in \mathscr{N}^{\perp}$ and $h \in \mathcal{N}$ (both not identically 0 ) such that

$$
\begin{gathered}
\left(I-\mu_{1} A\right) H=0, \\
S M\left(\beta^{*}\right) H-\mu_{1}^{-1} h=0 .
\end{gathered}
$$

But for $H \in \mathscr{N}^{\perp},(6.17)$ implies that $H=0$. Substituting $H=0$ in (6.18), we find that $h=0$. Thus, 0 cannot be an eigenvalue of $\mathbf{F}^{\prime}\left(0, b^{*}, \beta^{*}, 0\right)$, and $\mathbf{F}^{\prime}\left(0, b^{*}, \beta^{*}, 0\right)$ is invertible from $N^{\perp} \times \mathscr{N} \rightarrow \mathcal{N}^{\perp} \times \mathscr{N}$. The map, F, defined in (6.13)-(6.14) is analytic in $\Psi, b, \beta$, and $\tau$. Moreover, since $\mathbf{F}\left(0, b^{*}, \beta^{*}, 0\right)=\mathbf{0}$ and $\mathbf{F}^{\prime}\left(0, b^{*}, \beta^{*}, 0\right)$ is invertible, by the implicit function theorem [7, p. 26] we have the following. There exists a neighborhood of $\left(\beta^{*}, 0\right)$ in the form $\left(-\tilde{\rho}_{0}, \tilde{\rho}_{0}\right) \times\left(-\tilde{\tau}_{0}, \tilde{\tau}_{0}\right) \subseteq\left(-\rho_{0}, \rho_{0}\right) \times\left(-\tau_{0}, \tau_{0}\right)$ and there exist analytic functions, $b(\beta, \tau)$ and $\Psi(\beta, \tau)$, such that

(i) $\lim _{(\beta, \tau) \rightarrow\left(\beta^{*}, 0\right)} b(\beta, \tau)=b^{*}, \quad \lim _{(\beta, \tau) \rightarrow\left(\beta^{*}, 0\right)}\|\Psi(\beta, \tau)\|=0$,

(ii) $\mathbf{F}(\Psi(\beta, \tau), b(\beta, \tau), \beta, \tau)=\mathbf{0}$ for $(\beta, \tau) \in\left(-\tilde{\rho}_{0}, \tilde{\rho}_{0}\right) \times\left(-\tilde{\tau}_{0}, \tilde{\tau}_{0}\right)$. 
After substituting the solutions of $(6.15)$ (i.e., $\Psi(\beta, \tau)$ and $b(\beta, \tau))$ into $(6.14)$ and taking the inner product of the result with $z$, we obtain

$$
0=(M(\beta)(z+\Psi), z)+\tau^{-2}(R(\tau)(z+\Psi), z)-b(\beta, \tau) .
$$

Substituting (6.11) into (6.19) and simplifying, we obtain

$$
\begin{aligned}
b(\beta, \tau) & =(M(\beta) z, z)+(M(\beta) \Psi, z)+\tau^{-2}(R(\tau)(z+\Psi), z) \\
& =q \beta+2 c \beta^{2}+(M(\beta) \Psi, z)+\tau^{-2}(R(\tau)(z+\Psi), z) .
\end{aligned}
$$

From (6.12), we see that the sign of $b(\beta, \tau)$ determines the stability of $w(\beta, \tau)$. The values of $(\beta, \tau)$ for which the eigenvalues of $D(\beta, \tau)$ are zero are given by the solutions of

$$
b(\beta, \tau)=0 .
$$

From (6.20), it follows that there are two solutions of (6.21) at $\tau=0$. These are given by $(\beta, \tau)=\left(\hat{\beta}_{1}, 0\right)=(0,0)$ and $(\beta, \tau)=\left(\hat{\beta}_{2}, 0\right)=(-q /(2 c), 0)$. An implicit function theorem argument, similar to the one used in Section 5 , can be used to solve (6.21) in neighborhoods of $\left(\hat{\beta}_{1}, 0\right)$ and $\left(\hat{\beta}_{2}, 0\right)$, respectively. We omit the details and state the results. There exist positive constants, $\hat{\tau}_{1}$ and $\hat{\tau}_{2}$, and functions $\beta_{i}=\beta_{i}(\tau)$ such that for $i=1,2$,

(i) $\beta_{i}(\tau)$ is analytic on $|\tau|<\hat{\tau}_{i}$,

(ii) $\lim _{\tau \rightarrow 0} \beta_{i}(\tau)=\hat{\beta}_{i}$,

(iii) $b\left(\beta_{i}(\tau), \tau\right)=0$ for $|\tau|<\hat{\tau}_{i}$.

We define $\tau^{*}=\min \left\{\hat{\tau}_{1}, \hat{\tau}_{2}\right\}$.

Setting $\tau=0$ in (6.20), we observe the following (see Figure 6.1).

(i) $b(\beta, 0)=q \beta+2 c \beta^{2}>0$ when $\beta>\hat{\beta}_{1}$.

(ii) $b(\beta, 0)=q \beta+2 c \beta^{2}<0$ when $\hat{\beta}_{2}<\beta<\hat{\beta}_{1}$.

(iii) $b(\beta, 0)=q \beta+2 c \beta^{2}>0$ when $\beta<\hat{\beta}_{2}$.

For $|\tau|<\tau^{*}$, a sign change of $b(\beta, \tau)$ can only occur at $\beta=\beta_{1}(\tau)$ or $\beta=\beta_{2}(\tau)$. It follows that for all $|\tau|<\tau^{*}$,

(i) $b(\beta, \tau)>0$ for $\beta_{1}(\tau)<\beta<\tilde{\rho}_{0}$,

(ii) $b(\beta, \tau)<0$ for $\beta_{2}(\tau)<\beta<\beta_{1}(\tau)$,

(iii) $b(\beta, \tau)>0$ for $-\tilde{\rho}_{0}<\beta<\beta_{2}(\tau)$.

The stability of $w(\beta, \tau)$ is summarized in the following theorem.

THEOREM 2. Let $w(\beta, \tau)$ be a solution of $(*)$ in the form (6.7). Let $\beta_{1}(\tau)$ and $\beta_{2}(\tau)$ denote the solutions of $(6.21)$ for $|\tau|<\tau^{*}$. For every $|\tau|<\tau^{*}$ and $-\tilde{\rho}_{0}<\beta<\tilde{\rho}_{0}$,

(a) $w(\beta, \tau)$ is stable for $\beta_{1}(\tau)<\beta<\tilde{\rho}_{0}$,

(b) $w(\beta, \tau)$ is unstable for $\beta_{2}(\tau)<\beta<\beta_{1}(\tau)$,

(c) $w(\beta, \tau)$ is stable for $-\tilde{\rho}_{0}<\beta<\beta_{2}(\tau)$.

Remark. Suppose $w(\beta, \tau)$ is a solution satisfying (c) in Theorem 2 and that $\beta=$ $\hat{\beta}_{2}-\delta \in\left(-\tilde{\rho}_{0}, \beta_{2}(\tau)\right)$ with $\delta>0$. From (6.9) we have

$$
t(\beta, \tau)=-q^{2} /(4 c)+o(|\delta|)+o(|\tau|) .
$$

It follows that for $\delta>0$ and $\tau$ sufficiently small, $t(\beta, \tau)<0$. By Theorem 2 , the corresponding solution, $w(\beta, \tau)$, is subcritical and stable. 


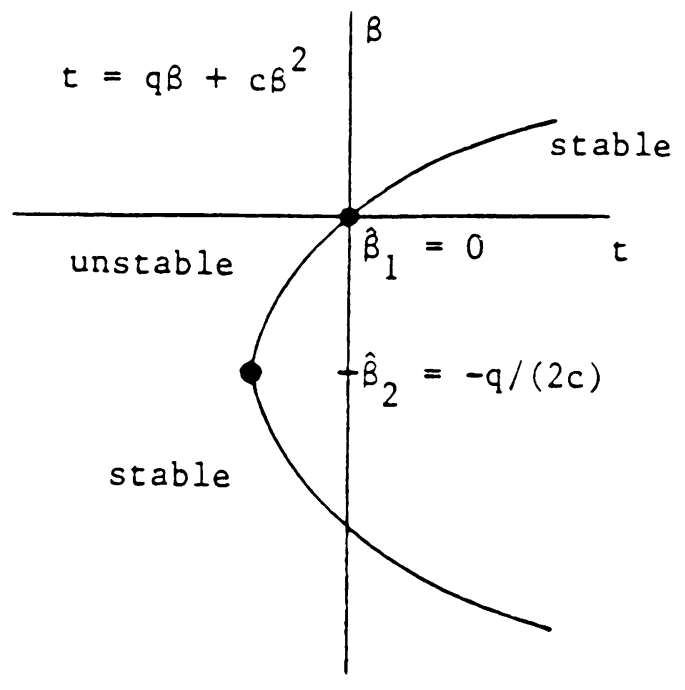

FIG. 6.1. Stability of solutions branching from the critical buckling load for a shallow cap in the $\tau=0$ plane.

7. Concluding remarks. It is easy to show that every classical solution of (2.31)(2.34) is a weak solution of (3.1)-(3.2) (or equivalently (3.10)-(3.11)). Moreover, every weak solution in the form (5.16) is also a classical solution. For if $w$ is a solution in the form (5.16), it is an analytic function of $u_{k}^{m}$. Since $u_{k}^{m}$ is smooth, the smoothness of $w$ follows.

We have shown that when the initial curvature, $K$, of the shell is small, one can introduce a new parameter, $\tau$ (proportional to $\sqrt{K}$ ), in addition to the usual load parameter, $\lambda$. The singularity at the branch point, $(w, \lambda)=\left(0, \mu_{1}^{0}+\tau^{2} / \mu_{1}^{0}\right)$, can then be unfolded in such a way to yield both subcritical and supercritical stable solutions.

Although it is not known whether the procedure discussed in Sections 4 and 5 will yield all solutions of small norm which branch from the trivial solution of $(*)$, we can say the following. Among those solutions in the form of (5.16), the axisymmetric solutions (given by (6.7)), are the only stable ones. This is due to the fact that for small $\tau$, the critical buckling load, $\lambda_{c}$, is of the form, $\lambda_{c}=\mu_{1}^{0}+\tau^{2} / \mu_{1}^{0}$, and the corresponding bifurcating solutions are axisymmetric. Hence, these solutions are also stable in $\mathscr{H}$. By a remark in Section 6, all other bifurcating solutions are unstable.

One should be cautious in attempting to extend these results to situations in which $K$ is not small, since the shallow cap approximation would no longer be valid.

Acknowledgment. The work presented here was part of the author's doctoral thesis which was completed under the direction of George Knightly at the University of Massachusetts. 


\section{LIST OF Symbols}

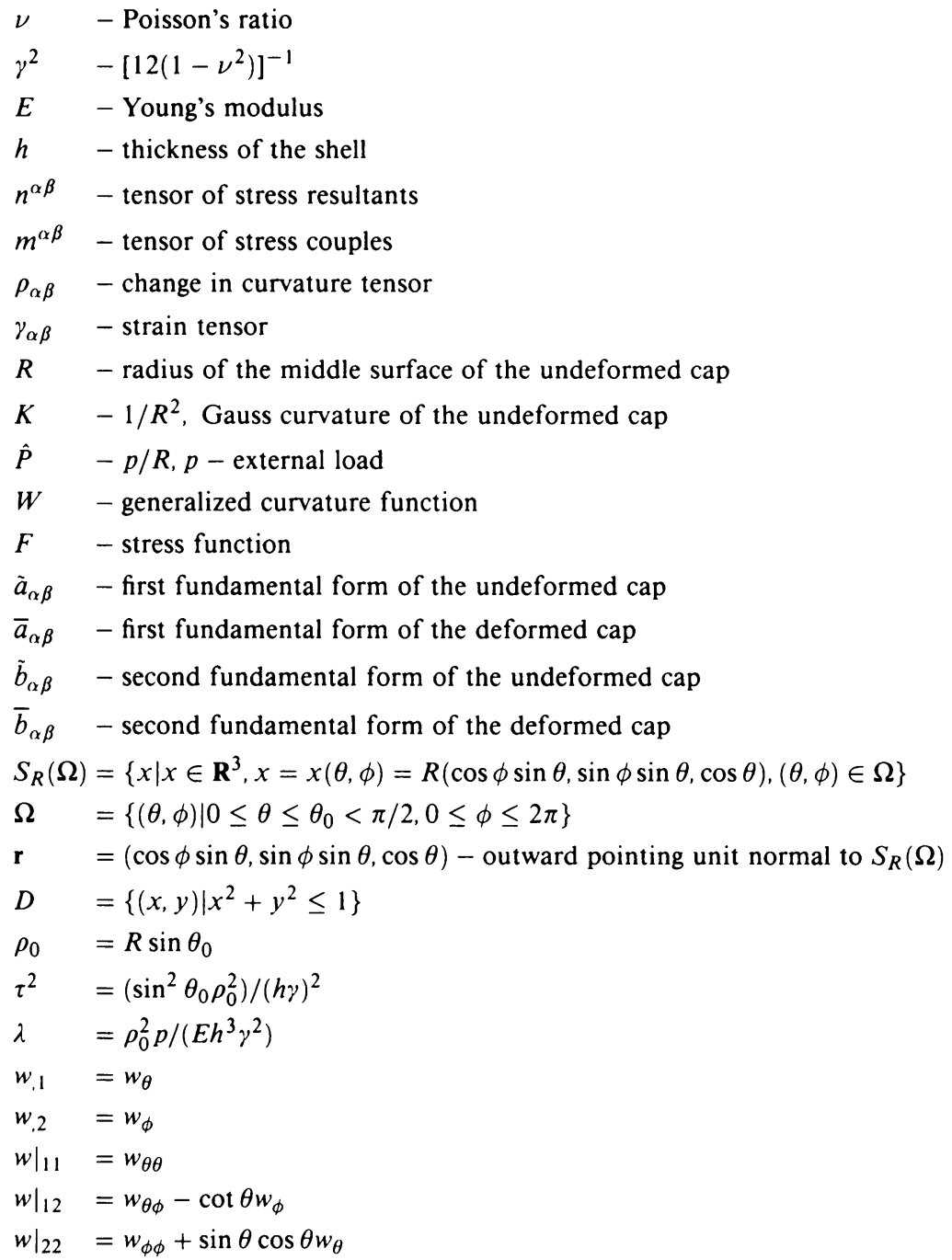

\section{REFERENCES}

[1] S. Agmon, Lectures on Elliptic Boundary Value Problems, Van Nostrand, Princeton, 1965

[2] L. Bauer, H. B. Keller, and E. L. Reiss, Axisymmetric buckling of hollow spheres and hemispheres, Comm. Pure Appl. Math. 23, 529-568 (1970)

[3] M. S. Berger, The existence of equilibrium states of thin elastic shells (I), Indiana Univ. Math. J. 20, 591-602 (1971)

[4] M. S. Berger and P. C. Fife, On Von Kármán's equations and the buckling of a thin elastic plate, Bull. Amer. Math. Soc. 72, 1006-1011 (1966)

[5] L. Berke and R. L. Carlson, Experimental studies of the postbuckling behavior of complete spherical shells, Experimental Mech. 8, 548-553 (1968)

[6] R. L. Carlson, R. L. Sendelbeck, and N. J. Hoff, Experimental studies of the buckling of complete spherical shells, Experimental Mech. 7, 281-288 (1967)

[7] S. Chow and J. Hale, Methods of Bifurcation Theory, Springer-Verlag, New York, 1982 
[8] P. Ciarlet and P. Rabier, Les équations de Von Kármán, Springer Mathematics Lecture Notes, Vol. 826, Springer-Verlag, Berlin, 1980

[9] F. John, Estimates for the derivatives of the stresses in a thin shell and interior shell equations, Comm. Pure Appl. Math. 18, 235-267 (1965)

[10] F. John, Refined interior equations for thin elastic shells, Comm. Pure Appl. Math. 24, 583-615 (1971)

[11] T. Kato, Perturbation Theory for Linear Operators, Springer-Verlag, Berlin/New York, 1963

[12] G. H. Knightly and D. Sather, Nonlinear axisymmetric buckled states of shallow spherical caps, SIAM J. Math. Anal. 6, 913-924 (1975)

[13] G. H. Knightly and D. Sather, Buckled states of a spherical shell under uniform external pressure, Arch. Rat. Mech. Anal. 72, 315-380 (1980)

[14] G. H. Knightly and D. Sather, Stable subcritical solutions for a class of variational problems, J. Differential Equations 46, 216-229 (1982)

[15] W. T. Koiter, The nonlinear buckling problem of a complete spherical shell under uniform external pressure, Proc. Kon. Nederl. Akad. Wet. Amsterdam, B72, 40-123 (1969)

[16] W. T. Koiter, On the nonlinear theory of thin elastic shells, Proc. Kon. Nederl. Akad. Wet. Amsterdam, B69, 1-54 (1966)

[17] J. B. McLeod and D. H. Sattinger, Loss of stability and bifurcation at a double eigenvalue, J. Functional Analysis 14, 62-84 (1973)

[18] W. Miller, Symmetry Groups and Their Applications, Academic Press, New York, 1972

[19] P. H. Rabinowitz, A note on topological degree for potential operators, J. Math. Anal. Appl. 51, 483-492 (1975)

[20] E. L. Reiss, Bifurcation buckling of spherical caps, Comm. Pure Appl. Math. 18, 65-82 (1965)

[21] D. Sather, Bifurcation and stability for a class of shells, Arch. Rat. Mech. Anal. 63, 295-304 (1977)

[22] D. H. Sattinger, Stability of bifurcating solutions by Leray-Schauder degree, Arch. Rat. Mech. Anal. 43, 154-166 (1971)

[23] C. Truesdell, Invariant and complete stress functions for general continua, Arch. Rat. Mech. Anal. 4, 1-29 (1959/60)

[24] G. N. Watson, Theory of Bessel Functions, Cambridge University Press, Cambridge, 1966

[25] H. J. Weinitschke, On asymmetric buckling of shallow spherical shells, J. Math. and Phys. 44, 141-163 (1965) 